\title{
Clinical systemic lupeol administration for canine oral malignant melanoma
}

\author{
INORU YOKOE $^{1^{*}}$, KAZUO AZUMA $^{1 *}$, KEISHI HATA $^{2}$, TOSHIYUKI MUKAIYAMA $^{3}$, TAKAHIRO GOTO ${ }^{3}$, \\ TAKESHI TSUKA ${ }^{1}$, TOMOHIRO IMAGAWA ${ }^{1}$, NORIHIKO ITOH $^{1}$, YUSUKE MURAHATA $^{1}$, \\ TOMOHIRO OSAKI $^{1}$, SABURO MINAMI $^{1}$ and YOSHIHARU OKAMOTO $^{1}$ \\ ${ }^{1}$ School of Veterinary Medicine, Faculty of Agriculture, Tottori University, Tottori 680-8553; ${ }^{2}$ Institute for Food and Brewing, \\ Akita Prefectural Agricultural, Forestry and Fisheries Research Center, Akita 010-1623; \\ ${ }^{3}$ Sakamoto Bio Co., Ltd., Yuuwa-Memeki, Akita 010-1233, Japan
}

Received June 13, 2014; Accepted July 15, 2014

DOI: $10.3892 / \operatorname{mco} .2014 .450$

\begin{abstract}
Canine oral malignant melanoma (COMM) is the most aggressive malignant tumor in dogs. Lupeol is a triterpene extracted from various fruits and vegetables that reportedly inhibits melanoma cell proliferation in vitro and in vivo. In this study, the efficacy of subcutaneous lupeol for spontaneous COMM was evaluated. A total of $11 \operatorname{dogs}(3$, 5 and 3 dogs diagnosed with clinical stage I, II and III melanoma, respectively) were evaluated. Subcutaneous lupeol $(10 \mathrm{mg} / \mathrm{kg}$ ) was administered postoperatively at various time points to treat these 11 COMM cases. Of the 11 subjects, 7 exhibited no local recurrence 180 days postoperatively and no severe adverse effects were observed in any of the cases. Furthermore, no distant metastasis was observed during the experimental period. Therefore, systemic lupeol may prevent local tumor progression and distant metastasis and may be a novel adjuvant treatment for the treatment of COMM.
\end{abstract}

\section{Introduction}

Canine oral malignant melanoma (COMM) is the most common malignancy in dogs, accounting for 30-40\% of all oral tumors $(1,2)$. COMM is characterized by extensive local invasion, as well as distant metastasis (2). Melanoma treatment includes radiation therapy and chemotherapy (CT), either alone, as an adjuvant therapy following surgery, or in combination (2). Although radiation therapy provides effective local tumor control, it is only performed in selected facilities (3-5).

Correspondence to: Professor Yoshiharu Okamoto or Dr Kazuo Azuma, School of Veterinary Medicine, Faculty of Agriculture, Tottori University, 4-101 Koyama Minami, Tottori 680-8553, Japan

E-mail: yokamoto@muses.tottori-u.ac.jp

E-mail:kazu-azuma@muses.tottori-u.ac.jp

*Contributed equally

Key words: canine, melanoma, lupeol, systemic injection, survival time
In addition, radiation therapy may be costly and, hence, beyond the financial means of some owners. Several reports have investigated the efficacy of CT in COMM, but there is no definitive evidence regarding its effectiveness (5,6,7-9). Therefore, there is a need for an alternative treatment for COMM.

Lupeol is a triterpene found in fruits such as olives, mangoes, strawberries, grapes and figs, numerous vegetables and several medicinal plants (10). Previous studies reported that lupeol has antitumor properties $(11,12)$ and several reports indicated that lupeol inhibits melanoma cell proliferation in vitro and in vivo $(13,14)$. Recently, Nitta et al $(14)$ reported that systemic lupeol administration inhibited tumor growth in a melanoma-bearing mouse model; however, there is no report evaluating its clinical efficacy in COMM. In this study, we aimed to evaluate the efficacy of subcutaneous lupeol as an adjuvant therapy for spontaneous COMM.

\section{Subjects and methods}

Subjects. A total of $11 \mathrm{dogs}$ were included in this study. The characteristics of the sublects are summarized in Table I. The represented breeds included 2 miniature Dachshunds, 2 Beagles, 2 miniature Schnauzers, 1 Golden Retriever, 1 Labrador Retriever, 1 American Cocker Spaniel, 1 Cavalier King Charles Spaniel and 1 mixed-breed dog. The dogs ranged in age between 8 and 17 years. The tumors in all 11 dogs were classified according to the TNM classification $(1,2)$ as follows: 3 dogs had stage I, 5 dogs had stage II and 3 dogs had stage III disease (Table I). Two cases of cancer recurrence were included (C04 and C07). This study was conducted between April, 2010 and March, 2013 in animal hospitals, including the Veterinary Teaching Hospital of Tottori University, Japan. Treatment was administered to dogs whose owners agreed to this study. The owners were informed of the risk of recurrence and other available treatment options, including surgery, radiation therapy and CT, and they all declined radiation therapy. The owners were offered the alternative treatment comprising surgery, subcutaneous lupeol and CT and they were all informed that lupeol was an experimental therapy. All the owners consented to the enrolment of their dogs in this clinical trial. 
Table I. Clinical case characteristics.

\begin{tabular}{llrrr}
\hline Case no. & \multicolumn{1}{c}{ Breed } & Age, years & Gender & Weight, kg \\
\hline C01 & Golden Retriever & 11 & Stage & 39 \\
C02 & Cavalier King Charles Spaniel & 17 & M & 7 \\
C03 & Labrador Retriever & 13 & I & I \\
C04 & Miniature Dachshund & 13 & M & I \\
C05 & Mixed-breed & 15 & F & II \\
C06 & Miniature Schnauzer & ND & M & 6 \\
C07 & Miniature Dachshund & 12 & II \\
C09 & Beagle & 8 & F & II \\
C10 & American Cocker Spaniel & 14 & M & 14 \\
C11 & Beagle & 12 & II \\
C12 & Miniature Schnauzer & 14 & II \\
\hline
\end{tabular}

M, male; F, female; ND, no data.

Table II. Clinical outcomes following melanoma therapy.

\begin{tabular}{|c|c|c|c|c|c|c|c|c|}
\hline $\begin{array}{l}\text { Case } \\
\text { no. }\end{array}$ & Stage & $\begin{array}{l}\text { Surgical } \\
\text { excision }\end{array}$ & $\begin{array}{c}\text { Combined } \\
\text { therapy }\end{array}$ & $\begin{array}{l}\text { Local } \\
\text { recurrence }\end{array}$ & $\begin{array}{c}\text { Distant } \\
\text { metastasis }\end{array}$ & DFT, days & ST, days & $\begin{array}{l}\text { Long-term } \\
\text { outcome }\end{array}$ \\
\hline $\mathrm{C} 01$ & I & Partial & - & - & - & 210 & 210 & Alive \\
\hline $\mathrm{C} 02$ & I & Partial & - & - & - & 210 & 210 & Alive \\
\hline $\mathrm{C} 03$ & I & Partial & - & Yes & - & 110 & 180 & Alive \\
\hline $\mathrm{C} 04$ & II & Partial & $\begin{array}{l}\text { Melphalan, } \\
\text { piroxicam }\end{array}$ & Yes & - & 170 & 186 & Alive \\
\hline $\mathrm{C} 05$ & II & Complete & - & - & - & 187 & 187 & Alive \\
\hline $\mathrm{C} 06$ & II & Partial & - & - & - & 180 & 180 & Alive \\
\hline $\mathrm{C} 07$ & II & Partial & PHCT & - & - & 120 & 420 & Alive \\
\hline $\mathrm{C} 08$ & II & Partial & - & - & - & 201 & 201 & Alive \\
\hline C09 & III & Partial & - & - & - & 248 & 248 & Alive \\
\hline $\mathrm{C} 10$ & III & Partial & PHT & - & - & 189 & 189 & Alive \\
\hline C11 & III & Partial & - & - & - & 39 & 39 & Alive \\
\hline
\end{tabular}

DFT, disease-free time; ST, survival time; PHCT, photodynamic hyperthermal chemotherapy; PHT, photodynamic hyperthermal therapy.

Surgical excision. Surgery was performed after the tumors were histopathologically confirmed as malignant melanomas. Complete excision was performed in 1 case (C05), in which the tumor could be excised with sufficient surgical margins. Partial excision without complete margins was performed in the remaining 10 cases; these tumors were difficult to excise completely due to their size or location (Table II).

Lupeol therapy. Lupeol solution was prepared as previously described (15). Briefly, lupeol was extracted from Indian lettuce (Lactua indica) and dissolved in olive oil (Wako Pure Chemical Industries Ltd., Osaka, Japan) using heat $\left(37^{\circ} \mathrm{C}\right)$ and sonification $(3 \mathrm{~h})$. The concentrated lupeol solution was diluted to $5 \mathrm{mg} / \mathrm{ml}$.

Lupeol was administered subcutaneously at $10 \mathrm{mg} / \mathrm{kg}$ as an adjuvant therapy at least 1 week postoperatively to prevent local recurrence or metastasis. Lupeol was initially administered twice a week for 2 weeks (4 administrations in total). Treatment was then decreased to once a week for a total of 4 weeks; alternate weeks for 8 weeks ( 4 administrations in total); once a month for several months ( $\geq 2$ administrations); and, finally, discontinued. However, this standard protocol was increased or decreased in frequency, depending on the owner's request, travel restrictions, or the physical condition of the subject. Follow-up examinations to detect recurrence and metastasis were performed once monthly during the trial period.

Combination therapy. Photodynamic hyperthermal therapy (PHT) $(16,17)$, photodynamic hyperthermal CT (PHCT) $(18,19)$ and CT were performed, with lupeol administered as an adjuvant therapy. PHT is a local treatment combining photo- 
dynamic therapy and hyperthermia that uses indocyanine green as a photosensitizer $(16,17)$. PHCT is an advanced form of PHT, in combination with carboplatin CT $(18,19)$.

\section{Results}

Clinical outcome. The clinical outcomes are summarized in Table II. There were no severe adverse effects, such as local pain, diarrhea, or vomiting, in any of the subjects during lupeol treatment. Local tumor recurrence occurred in 1 case with stage I disease (C03) at 110 days and in 1 case with stage II disease (C04), which underwent partial surgical excision initially, at 170 days. Case $\mathrm{C} 03$ was immediately restarted on lupeol therapy alone every 2 weeks. After the additional treatment, tumor progression was arrested in case $\mathrm{C} 03$; and the disease condition has remained stable in both cases. In case $\mathrm{C03}$, the tumor decreased in size and the owner reported that the dog's quality of life had improved.

Survival. At the end of the experimental period, all the dogs remained alive. Of the $12 \mathrm{dogs}, 10$ had survived for $>180$ days after surgery. Moreover, no distant metastasis was observed during the experimental period.

\section{Discussion}

COMM is an extremely malignant tumor, with a high propensity for local invasion and metastasis $(1,2)$. The treatment options for COMM include surgery, radiation therapy, CT and immunotherapy comprising xenogeneic DNA vaccination. MacEwen et al (20) reported that the median survival time (ST) in dogs with oral malignant melanoma that underwent complete surgical excision was $\sim 17-18$ months, 5-6 months and 3 months for stage I, II and III disease, respectively. There may be a potential to prolong ST beyond these reported durations, as all the subjects remained alive during the most recent examination. In stage III cases, the median ST increased in subjects administered adjuvant lupeol treatment ( $>6$ vs. 3 months). The reported median ST for dogs receiving radiotherapy ranges between 5.3 and 11.9 months $(3-5,7,9)$; however, as the radiotherapy equipment, treatment protocols and supplementary therapies differ between studies, it is difficult to directly compare previously reported results. Regardless, the results of previous reports suggest that systemic lupeol administration may help prolong the median ST in COMM cases and may prove efficacious as an adjuvant postoperative treatment.

Distant metastasis was not observed in any of the cases, including the 3 stage III cases, which were at highest risk for metastasis. Furthermore, there was no local recurrence for 180 days postoperatively in 7 of the $11 \mathrm{dogs}$. The median disease-free time (DFT) of dogs undergoing radiation therapy reportedly ranges between 5 and 7.9 months $(5,20)$. In a study evaluating radiation therapy in 38 dogs with COMM, Theon et al (21) reported a median DFT of 11.3 months in stage T1 cases, 6.0 months in stage T2 cases and 6.7 months in stage T3 cases. The results of the present study suggest that surgery combined with lupeol administration achieves local tumor control equivalent to that of radiation therapy. Saleem et al (13) reported that lupeol inhibits the growth of highly aggressive human metastatic melanoma cells in vitro and in vivo by inducing apoptosis. The results of the present study suggest that, in addition to preventing local progression, systemic postoperative adjuvant lupeol administration may also prevent the development of distant tumor metastasis.

For the local treatment of COMM, aggressive complete surgical excision, such as partial mandibulectomy and maxillectomy is the most effective option (5). Radiation therapy also plays an important role and is an effective treatment for achieving local tumor control; however, radiation therapy is associated with several adverse effects, including dermal desquamation, alopecia and bone necrosis, which may negatively affect the quality of life (7). The subjects must be anesthetized during radiation therapy, which itself is accompanied by serious risks. In addition, therapy is only available at selected facilities and may not be affordable for all owners; therefore, radiation therapy cannot be easily performed in all cases. CT combined with radiation therapy and surgery has been used as an alternative systemic therapy to prevent local recurrence and distant metastasis. Although several reports have evaluated the efficacy of CT in COMM as a sole or adjuvant therapy, there is no clear evidence that $\mathrm{CT}$ decreases the risk of local recurrence or metastasis, or that it prolongs median ST (5-9). These limitations highlight the need to develop an effective systemic therapy for COMM.

PHT, PHCT, melphalan and piroxicam were administered in combination, but there is no evidence that these treatments are effective in controlling COMM progression. PHT and PHCT were performed with owner consent after explaining the experimental nature of these treatments. Proulx et al (5) reported that melphalan administered for COMM exerted no beneficial effects. There is limited evidence that non-steroidal anti-inflammatory drugs (NSAIDs) may exert a protective effect against malignant melanoma development in humans (22); however, in a study evaluating NSAID therapy in dogs, there was no significant difference in survival attributed to NSAID administration and NSAID therapy did not provide any survival benefit or achieved an improved therapeutic response in the treated subjects (7). Therefore, these adjuvant therapies were unlikely to confer any beneficial treatment effect in this study.

No severe adverse effects, such as local pain, were observed in any of the dogs during lupeol treatment. There are no known reports of adverse effects associated with lupeol administration, which indicates that lupeol is safe when administered at the dosage recommended in the present study. Notably, this study was only a limited clinical trial and evaluated lupeol as a postoperative adjuvant therapy. Prospective controlled studies are required to assess the clinical efficacy of subcutaneous lupeol administration for the treatment of COMM, as well as in combination with other modalities, such as radiotherapy and CT.

In conclusion, to the best of our knowledge, this study is the first clinical trial to evaluate systemic lupeol therapy for COMM. Postoperative lupeol is easily administered, is not associated with severe adverse effects and was shown to prevent local tumor progression and distant metastasis. Therefore, the results of the present study suggest that lupeol is a potential novel adjuvant treatment option and may be used as systemic therapy for COMM. 


\section{References}

1. Smith SH, Goldschmidt MH and McManus PM: A comparative review of melanocytic neoplasms. Vet Pathol 39: 651-678, 2002.

2. Bergman PJ: Canine oral melanoma. Clin Tech Small Anim Pract 22: 55-60, 2007.

3. Bateman KE, Catton PA, Pennock PW and Kruth SA: 0-7-21 radiation therapy for the treatment of canine oral melanoma. J Vet Intern Med 8: 267-272, 1994.

4. Blackwood L and Dobson JM: Radiotherapy of oral malignant melanomas in dogs. J Am Vet Med Assoc 209: 98-102, 1996.

5. Proulx DR, Ruslander DM, Dodge RK, Hauck ML, Williams LE, Horn B, Price GS and Thrall DE: A retrospective analysis of 140 dogs with oral melanoma treated with external beam radiation. Vet Radiol Ultrasound 44: 352-359, 2003.

6. Rassnick KM, Ruslander DM, Cotter SM, Al-Sarraf R, Bruyette DS, Gamblin RM, Meleo KA and Moore AS: Use of carboplatin for treatment of dogs with malignant melanoma: 27 cases (1989-2000). J Am Vet Med Assoc 218: 1444-1448, 1996.

7. Murphy S, Hayes AM, Blackwood L, Maglennon G, Pattinson H and Sparkes AH: Oral malignant melanoma - the effect of coarse fractionation radiotherapy alone or with adjuvant carboplatin therapy. Vet Comp Oncol 3: 222-229, 2005

8. Dank G, Rassnick KM, Sokolovsky Y, Garrett LD, Post GS, Kitchell BE, Sellon RK, Kleiter M, Northrup N and Segev G: Use of adjuvant carboplatin for treatment of dogs with oral malignant melanoma following surgical excision. Vet Comp Oncol 12: 78-84, 2014

9. Freeman KP, Hahn KA, Harris FD and King GK: Treatment of dogs with oral melanoma by hypofractionated radiation therapy and platinum-based chemotherapy (1987-1997). J Vet Intern Med 17: 96-101,2003.

10. Saleem M, Kaur S, Kweon MH, Adhami VM, Afaq F and Mukhtar H: Lupeol, a fruit and vegetable based triterpene, induces apoptotic death of human pancreatic adenocarcinoma cells via inhibition of Ras signaling pathway. Carcinogenesis 26: 1956-1964, 2005.

11. Chaturvedi PK, Bhui K and Shukla Y: Lupeol: connotations for chemoprevention. Cancer Lett 263: 1-13, 2008.

12. Saleem M: Lupeol, a novel anti-inflammatory and anti-cancer dietary triterpene. Cancer Lett 285: 109-115, 2009.
13. Saleem M, Maddodi N, Abu Zaid M, Khan N, bin Hafeez B, Asim M, Suh Y, Yun JM, Setaluri V and Mukhtar H: Lupeol inhibits growth of highly aggressive human metastatic melanoma cells in vitro and in vivo by inducing apoptosis. Clin Cancer Res 14: 2119-2127, 2008.

14. Nitta M, Azuma K, Hata K, Takahashi S, Ogiwara K, Tsuka T, Imagawa T, Yokoe I, Osaki T, Minami S and Okamoto Y: Systemic and local injections of lupeol inhibit tumor growth in a melanoma-bearing mouse model. Biomed Rep 1: 641-645, 2013.

15. Hata K, Hori K, Mukaiyama T, Sakamoto K and Takahashi S Activators of melanin biosynthesis from Lactuca indica. Nat Med 57: 238-241, 2003.

16. Radzi R, Osaki T, Tsuka T, Imagawa T, Minami S, Nakayama Y and Okamoto Y: Photodynamic hyperthermal therapy with indocyanine green (ICG) induces apoptosis and cell cycle arrest in B16F10 murine melanoma cells. J Vet Med Sci 74: 545-551, 2012

17. Radzi R, Osaki T, Tsuka T, Imagawa T, Minami S and Okamoto Y: Morphological study in B16F10 murine melanoma cells after photodynamic hyperthermal therapy with indocyanine green (ICG). J Vet Med Sci 74: 465-472, 2012.

18. Onoyama M, Tsuka T, Imagawa T, Osaki T, Minami S, Azuma K, Kawashima K, Ishi H, Takayama T, Ogawa N and Okamoto Y: Photodynamic hyperthermal chemotherapy with indocyanine green: a novel cancer therapy for 16 cases of malignant soft tissue sarcoma. J Vet Sci 15: 117-123, 2014.

19. Onoyama M, Azuma K, Tsuka T, Imagawa T, Osaki T, Minami S, Ogawa N and Okamoto Y: Effects of photodynamic hyperthermal therapy with indocyanine green on tumor growth in a colon 26 tumor-bearing mouse model. Oncol Lett 7: 1147-1150, 2014

20. MacEwen EG, Patnaik AK, Harvey HJ, et al: Canine oral melanoma: comparison of surgery versus surgery plus Corynebacterium parvum. Cancer Invest 4: 397-402, 1986.

21. Theon AP, Rodriguez C and Madewell BR: Analysis of prognostic factors and patterns of failure in dogs with malignant oral tumors treated with megavoltage irradiation. J Am Vet Med Assoc 210: 778-784, 1997.

22. Boria PA, Murry DJ, Bennett PF, Glickman NW, Snyder PW, Merke BL, Schlittler DL, Mutsaers AJ, Thomas RM and Knapp DW: Evaluation of cisplatin combined with piroxicam for the treatment of oral malignant melanoma and oral squamous cell carcinoma in dogs. J Am Vet Med Assoc 224: 388-394, 2004. 